Voix et Images

voixetimages

\title{
Excursions dans les univers de la science-fiction
}

\section{Christian Vandendorpe}

Volume 26, numéro 1 (76), automne 2000

\section{L'immonde}

URI : https://id.erudit.org/iderudit/201525ar

DOI : https://doi.org/10.7202/201525ar

Aller au sommaire du numéro

\section{Éditeur(s)}

Université du Québec à Montréal

ISSN

0318-9201 (imprimé)

1705-933X (numérique)

Découvrir la revue

Citer cet article

Vandendorpe, C. (2000). Excursions dans les univers de la science-fiction. Voix et Images, 26(1), 173-177. https://doi.org/10.7202/201525ar d'utilisation que vous pouvez consulter en ligne.

https://apropos.erudit.org/fr/usagers/politique-dutilisation/ 


\title{
Excursions dans les univers de la science-fiction
}

\author{
Christian Vandendorpe, Université d'Ottawa
}

La science-fiction n'a jamais réussi à se tailler sa place au soleil de l'institution littéraire. Reléguée dans les marges, elle est encore le plus souvent considérée comme un genre pour adolescents, dont il y aurait finalement peu à dire. Si, dès les années soixante, le fantastique atteignait à la respectabilité avec l'ouvrage charnière de Todorov, la science-fiction est restée le parent pauvre du trinôme générique "merveilleux, fantastique et sciencefiction". Todorov n'y consacrait d'ailleurs que quelque lignes, en y voyant un simple cas de "merveilleux scientifique ". Il faut dire en outre que le genre s'est surtout développé en langue anglaise et qu'il est presque impossible d'en parler sans faire référence aux noms d'Asimov, Herbert, Dick, Gibson et bien d'autres, même si la production française mérite aussi qu'on s'y arrête.

Ce halo négatif et l'hétérogénéité linguistique du corpus n'ont pas ar- rêté Richard Saint-Gelais, dont L'empire du pseudo apporte à la connaissance du genre une contribution non négligeable ${ }^{1}$. Son objectif n'est rien moins que de "repenser à nouveaux frais la place de la science-fiction dans l'échiquier mobile de la fiction contemporaine" (p. 12). Il ne faut donc pas s'attendre à une nouvelle recension des thèmes caractéristiques du genre, comme l'ont fait J. van Herp (1973), les frères Bogdanoff (1976), ou, sur un plan typologique, G. Bouchard (1993). On n'y trouvera pas davantage une réflexion sur l'essence du genre, comme s'y était attaché un collectif québécois dont SaintGelais semble curieusement ignorer l'existence ${ }^{2}$. Bien au contraire, cette dernière approche devrait être irrémédiablement abandonnée:

L'hypothèse qui sous-tend cet essai est qu'une compréhension du phénomène générique passe par l'abandon d'une conception essentialiste qui aboutirait à une hypostase des genres, en y voyant 
une matrice ou un ensemble de propriétés plutôt qu'un domaine de pratiques. (p. 12-13)

Il existe toutefois des "motifs structurels - récurrents, qui ont présidé à la constitution du genre, et ceux-ci sont examinés dans la première partie. Le plus évident est le récit d'anticipation, qui rompt avec le point de vue habituel du récit rétrospectif. Comment raconter des faits censés se dérouler dans le futur? Une telle question peut paraître oiseuse aujourd'hui, où l'on recourt naturellement à un narrateur qui raconte à partir d'un futur encore plus lointain que celui des événements racontés. Mais, avant d'en arriver là, il a fallu accréditer le procédé auprès des lecteurs. Divers critiques ont ainsi mis en évidence des exemples de balbutiements, qu'il s'agisse de cas d'alternance maladroite entre le présent et le futur chez Restif de la Bretonne ou d'une lourde insistance sur le moment futur de la narration chez Jules Verne. Quoi qu'il en soit, le récit d'anticipation, s'il réussit rarement à prévoir correctement un état du futur, propose toujours, en creux, une image plus ou moins détaillée du moment où il a été écrit et de l'imaginaire de son époque. Sous sa forme naïve la plus courante, l'anticipation présente une "réduplication appauvrie de la réalité quotidienne" (Michel Butor), voire des états afossiles de la pensée" (Brian Aldiss). Pour SaintGelais, il ne faudrait pas s'en offusquer, les jugements des lecteurs étant eux-mêmes toujours inscrits dans une historicité, au même titre que les anticipations sur lesquelles ils portent.

L'uchronie est un autre des motifs structurels identifiés par SaintGelais. Elle consiste à "proposer un monde fictif qui ne relève ni du futur ni du passé au sens strict, mais plutôt d'une Histoire qui aurait pris un cours différent de celui qu'elle a pris en réalité" (p. 43). Les exemples invoqués sont variés et intéressants, allant de François Hertel à Norman Spinrad, en passant par Alain Bergeron, Geoffroy, Renouvier, l'incontournable Philip K. Dick et bien d'autres. À tel point qu'on est un peu surpris de ne pas y trouver le maître actuel du genre, l'Italien Valerio Evangelisti, qui a créé le fascinant personnage de Nicolas Eymerich. Préoccupé par les rapports entre la fiction et le "réel , Saint-Gelais met en parallèle les récits uchroniques et l'Histoire conjecturale, pour décréter, comme on s'y attendait, que ces genres fort similaires diffèrent surtout par les "réglages de lecture "qu'ils suscitent chez le lecteur. On peut toutefois s'interroger sur l'extension donnée au terme "fiction", qui désigne dans cet ouvrage aussi bien les récits imaginaires que les hypothèses et spéculations du lecteur, quand bien même ce dernier ne les "considère pas comme telles" (p. 64). Cette position permet à Saint-Gelais de placer dans le champ de la "fiction" à la fois un récit de Philip K. Dick et la critique qui en a été faite par Darko Suvin, laquelle serait une "fiction inavouée": à voir effacée ainsi toute distance entre la lecture critique et son objet, tout comme entre le texte et la pensée, on en arrive à se demander si les opinions de Saint-Gelais ne relèveraient pas, elles aussi, de la fiction.

Parmi les autres motifs structurels étudiés, il y a évidemment les jeux avec le temps, constitutifs du genre, que l'auteur aborde à l'aide de quelques notes disparates et rapides 
sur la vitesse. Une plus grande attention est accordée aux "détections science-fictionnelles", c'est-à-dire aux histoires de détective, pour l'analyse desquelles l'auteur mobilise sa connaissance du genre policier. Au terme de son enquête, il reconnaît deux façons de combiner sciencefiction et énigme policière, soit en intégrant une "énigme restreinte" à un monde étranger, selon un modèle bien rodé depuis Asimov, soit en faisant "du texte tout entier, avec ses entrelacs et ses replis secrets, le terrain de l'enquête" (p. 129).

Dans une deuxième partie, SaintGelais met en évidence les caractéristiques de l'imaginaire sciencefictionnel et ce qui le distingue de la fiction réaliste. Pour ce faire, il prend comme point d'entrée la place faite au didactisme, qui est la façon la plus classique de naturaliser l'étrange. Après une première époque où la science-fiction recourait volontiers au didactisme - qu'on se souvienne de Jules Verne et de ses extraits d'encyclopédie - , on en est venu à poser d'entrée de jeu des réalités imaginaires dont les seules dénominations ont pour effet de plonger dans la perplexité un lecteur habitué à trouver dans son "encyclopédie "les référents de tous les termes rencontrés. Ce jeu sur les "paradigmes absents", selon l'expression de Marc Angenot, s'est accentué dans les années 19401960. La révolution "New Wave" qui survient ensuite "tournera carrément le dos à la transparence du discours didactique au profit d'une opacité narrative marquée" (p. 167).

Un des points forts de cet ouvrage consiste à mettre en évidence certaines des conventions de lecture propres au champ de production science-fictionnel. On admettra volontiers la nécessité de prendre en compte une telle dimension, facilement oubliée par les approches "positives " centrées sur une "ontologie " du genre. Mais l'auteur veut tellement prouver qu'il pousse le bouchon un peu loin, au risque de perdre l'adhésion de ses lecteurs. Ainsi affirme-t-il le plus sérieusement du monde que "si Madame Bovary ou $\dot{A}$ la recherche du temps perdu ne sont pas considérés comme des romans de science-fiction, c'est qu'ils ne sont pas lus comme tels" (p. 231). Aucun exemple ne vient étayer cette affirmation en ce qui concerne l'ouvrage de Flaubert.

Au sujet de Proust, l'auteur invoque le fait que plusieurs noms de lieu n'ont pas de référents ou que certains passages dénotent, de la part du narrateur, une véritable captation des pensées d'un autre personnage ou, encore, qu'une métaphore sur les vertèbres de la tante Léonie oblige à conclure que celles-ci seraient mal placées. Voilà qui est bien maigre et fort injuste pour la pauvre Léonie! Loin d'étayer la thèse, cette discussion spécieuse tend à confirmer indirectement le statut science-fictionnel de certains traits, tels le recours à des lieux imaginaires, la télépathie et les variations anatomiques: preuve qu'il est difficile d'échapper à l'essentialisme! Par ailleurs, on aimerait que soient davantage précisés l'activité de lecture et ces fameux "réglages " qui font que tel texte sera lu comme de la science-fiction et tel autre comme un récit réaliste: par quels mystérieux processus ces réglages sont-ils mis en place? La dimension proprement cognitive ne devrait-elle pas être considérée, comme elle l'était 
déjà chez Todorov, qui voyait l'essence du fantastique dans l'hésitation éprouvée devant des événements en apparence surnaturels? Ne faudrait-il pas prendre en compte les effets du texte sur le lecteur, par exemple au plan des émotions engendrées : terreur, curiosité, étonnement? Après tout, bien des collections mettent de tels traits en évidence et les récits de lâge d'or de la science-fiction américaine étaient présentés comme des "wonder stories" ou des "amazing stories". Enfin, ne serait-il pas légitime de faire intervenir l'activité catégorisante spontanée au moyen de laquelle le cerveau tend à regrouper ses expériences et à les synthétiser en vue de décisions futures? Bref, à moins de verser dans le subjectivisme intégral, il semble bien que les réglages de lecture ne soient pas séparables du matériau textuel qui en a permis la constitution. Par ailleurs, on s'étonne que Saint-Gelais n'ait pas jugé utile d'appuyer sa réflexion sur la science-fiction en confrontant celle-ci à des genres voisins, comme le merveilleux et le fantastique, auxquels il daigne à peine consacrer une note en bas de page, alors que ces genres produisent des réglages de lecture différents.

Une vue plus générale de l'ensemble du champ lui aurait également évité d'élargir le concept de métafiction au point où ce dernier perd toute valeur critique. On sait que, pour Patricia Waugh ou Linda Hutcheon, la métafiction se définit comme une fiction qui est aussi une critique, impliquant de la part de l'auteur une posture réflexive sur le récit en cours. Selon Saint-Gelais, une telle posture apparaîtrait dès que l'illusion s'intensifie “jusqu'au point où elle s'autodénonce" (p. 258). Ainsi croit-il pouvoir affirmer que "la science-fiction a été un genre métafictionnel, et ce dès ses débuts " parce que les réglages de lecture qu'elle met en place sont très différents de " ceux qui caractérisent la lecture réaliste " (p. 268). Mais alors, en vertu de ce raisonnement, le merveilleux des Mille et une nuits devrait aussi appartenir de plein droit à la métafiction... En outre, une telle façon d'opposer la science-fiction à la fiction réaliste - même si ce dernier terme est souvent mis entre guillemets relève d'une dichotomie commode et simpliste. Le réalisme n'est en effet qu'une des provinces du régime fictionnel, à côté duquel coexistent des domaines immenses, tels le fantastique, le merveilleux ou l'allégorie.

On trouve un autre exemple de cette myopie théorique dans le dernier chapitre, consacré à "la constellation Star Trek". Examinant le prodigieux succès de cette série télévisée, l'auteur semble s'étonner des multiples produits qui en sont dérivés (films, dessins animés, novélisations, fanzines, dictionnaire Klingon, etc.) et il voit dans ce "multivers" un phénomène qui serait en train " de modifier de fond en comble l'économie traditionnelle de la fiction ", dont les univers auraient traditionnellement été "parfaitement clos" (p. 359). Mais le phénomène est-il si nouveau? En fait, on pourrait soutenir, beaucoup plus justement, que le régime de la fiction populaire est essentiellement ouvert, ce qui permet le phénomène des séries et des produits dérivés. Bien avant notre époque, la mythologie grecque avait développé un vaste ensemble de récits qui ont été inlassablement repris, augmentés, inter- 
prétés et représentés sous toutes les formes possibles depuis plus de trois millénaires. Il en va de même de la chanson de gestes médiévale. Et la science-fiction tire précisément son attrait du fait qu'elle recycle des matériaux qui font rêver l'humanité depuis la nuit des temps.

Cela dit, l'ouvrage de Saint-Gelais n'en constitue pas moins une réflexion intéressante sur le genre, étoffée de nombreux exemples. Au plan de la présentation, le lecteur devra surmonter l'agacement produit par un choix discursif qui oblige à alterner constamment entre le corps du texte et les notes en bas de page, ce qui ralentit considérablement la lecture. Alors que le recours au système MLA aurait normalement dû entraîner leur disparition, des bataillons de notes capitonnent chaque pied de page, apportant le plus souvent des commentaires ou des éléments de discussion arbitrairement détachés du texte principal. Enfin, une normativité pointilleuse souligne par des sic d'hypothétiques défauts de langage, tel l'emploi de "ceci * au lieu de "cela * (?) dans une citation de Perec (p. 150, note 22). On aurait pu espérer que l'auteur transcenderait ce genre de détail (et non "transcendrait", sic, p. 69), surtout quand il s'agit d'un choix stylistique qui ne nuit aucunement au mouvement de la lecture.

1. Richard Saint-Gelais, L'empire du pseudo. Modernités de la science-fiction, Québec, Nota Bene, 1999, 399 p.

2. Aurélien Boivin, Maurice Emond, Michel Lord, Les ailleurs imaginaires, Québec, Nuit Blanche, $1993,306 \mathrm{p}$. 